\title{
METHODOLOGY FOR EMPIRICAL RESEARCH ON BENCHMARKING APROACH IN BULGARIAN FIRMS
}

\author{
Milena Kirova ${ }^{1}$, Irina Petrova ${ }^{2}$ \\ Faculty of Business and Management, University of Ruse, 8 Studentska str., Ruse, Bulgaria \\ E-mails: ${ }^{1}$ mkirova@uni-ruse.bg; ${ }^{2}$ impetrova@uni-ruse.bg (correspondingauthor)
}

Received 09 April 2020; accepted 05 May 2020

\begin{abstract}
The main purpose of this article is to demonstrate the methodology of empirical research to evaluate the degree of awareness and implementation of benchmarking by Bulgarian manufacturing companies. To achieve this goal, the authors have set the following tasks: First to identify the companies that fall into the target group. Second, to collect primary data through a survey of the management of the companies included in the target group. Third, to conduct a semi-structured interview with management team representatives in order to gather expert opinions. The method of partial statistical survey of a general population was used by studying a representative sample formed with the help of non-repetitive selection of units. The results of the survey will be the basis for a new approach in the development of company strategies based on benchmarking analysis.
\end{abstract}

Keywords: benchmarking, methodology, empirical research, innovation, strategy.

JEL Classification: C10, L11, L21, M11, O14, O30.

\section{Introduction}

The speed of increasing the uniqueness of products and services, the intensively changing requirements of the market and the ever growing pressure on the part of society are the trends, which force the companies to introduce new practices, in order to preserve their competitiveness. In the future, only those companies will survive, which manage to create knowledge and are ready to learn from the experience of others (Boneva, 2018). Innovation will be the cornerstone of organisational culture, and experimentation will be stimulated in many and different ways (Ghinea, Mihaylova, \& Papazov, 2015). In order to continue their existence in an environment, characterised by increasing turbulence, the companies will be based on their core competences and their sustainable competitive advantages (Kostadinova \& Antonova, 2018). An important factor for the success of a certain company is its skill to manage the innovations and the processes related to them, or the so called innovation management (Kirova, 2009). One of the main approaches, used in innovation management could be a benchmarking for developing innovation strategies.

\section{Literature review}

At its core, benchmarking is a tool for analysis and evaluation of the company's activity and it facilitates the transfer of management approaches and methods for using the experience and good practices of the units and organisations we compare to (Marinov, Vitliemov, \& Popova, 2017). Practically, benchmarking is accepted as a measure or standard for the highest achievements and serves as a benchmark against the best companies in a specific field (Simeonova \& Nedyalkov, 2019). For this very reason it is becoming a way of analysing and enhancing the competitive advantages of the company, i.e. a metrics instrument, but at the same time a structured approach to achieving high results, which lead to increased benefits for the customers and society (Kirova, 2011). Although initially benchmarking was directed mainly towards learning about other companies' products and services, its sphere gradiually expanded and included work processes, staff functons, company performance and the whole process of providing value (Pavlov, Sheresheva, \& Perello, 2019).

As a result of reviewing the literature, it can be concluded that (Chiprianov, 2008):

- Applying benchmarking and implementing the experience of the best should be used as an opportunity for evaluation of the company's own level, for obtaining a clear and objective idea about the strengths and weaknesses, for the proper identification of the objects to be im- 
proved and for developing and applying a programme for improving inneficent activities and processes;

- The benefits of applying benchmarking to company practice show that it is strategically needed for their further development;

- A study of the literature showed the lack or insufficiency of information on the application of benchmarking as an innovative approach in the Bulgarian practice;

- To prove the urgency of the problem, namely, the insufficient use of benchmarketing as an approach for innovative management, the authors will carry out an empirical study on the degree of using this approach by the Bulgarian companies;

- In this regard the authors develop a methodology which can be used for implementing the above empirical study.

\section{The methodology}

\subsection{Main goal of the research}

Assessing the degree of awareness and implementation of benchmarking approach by the Bulgarian manufacturing medium and large companies.

\subsection{The methodology}

Own research by conducting:

- A survey among medium and large companies in the region of Ruse from different production fields (for comparative analysis) (Conev, 1968);

- A semi-structured interview among employees at different levels of management in the company (Chengelova, 2013);

For solving the research goal and objectives a plan has been developed, including several stages:

- Stage one - identifying the companies of the target group (Gatev, 1995);

- Stage two - collecting primary data through a survey, directed to the management staff of the target group companies (Nedyalkov, 2011);

- Stage three - conducting a semi-structured interview with representatives of management teams, in order to collect expert opinions (The Global Benchmarking Network, 2010).

In this paper the authors review the process of determining the size of the sample, the structure and content of the questionnaire and the nonstructured interview in details.

\section{Identifying the companies in the target group - stage one}

Studying the social and economic phenomena and processes, it is not always recommendable and economically viable to study all the units of the aggregate. In practice, more and more often the institutions and the independent researchers prefer to take into consideration only part of the units. This means that the studies are conducted,based on the analysis of a small part of the aggregate (Pavlov, 2013).

In theory and practice, statistic research is comprehensive or partial. The representative statistical study is one of the most sophisticated and scientifically substanciated studies (Kostov \& Todorov, 1999). This study focuses only on some of the units, but their choice and number provide the necessary representativeness and reliability of results for the units from the aggregate.

There are two types of aggregates with the representative study, namely general (total, comprehensive) and representative sample. The main objective of this type of studies is to assess the values for the general aggregate, which are unknown, on the basis of the summarising characteristics revealed.

To make the estimates of the general aggregate of empirical studies reliable and substantiated, it is necessary the estimates themselves and the samples to answer certain requirements, namely:

a) the samples should be randomly generated, i.e. they should be random;

b) to contain the large number of units needed and to make it impossible for a unit already chosen to be replaced by another (Saikova \& Todorova, 1994).

The objective for each researcher, conducting a representative study is to find accurate estimates of the aggregate parameters that are satisfactory. These are proportion, aberration, mean values, etc. To achieve this, stochastic or representative samples should be used, or, in other words, random samples.

To devise random samples, different methods are used:

- mechanical (systematic) selection;

- regional (typical) selection;

- serial (nest) selection;

- random (lottery) selection: the random selecteion can be repeatable and nonrepeatable. With the repeatable selection each unit selected can be "returned" to the general aggregate and participate in the generation of the sample again. When we 
speak about non-repeatable method of sample generation, there if the unit has been included in the sample once, it cannot participate in its generation any more.

The way a random sample is completed determines its type: mechanical (systematic) sample, typical sample, nest sample, combined sample or random (stochastic) sample.

In order to reach the objectives set for this study and based on theory and practice, the authors will conduct partial statistical survey of a general aggregate, which is the object of the study, through studying and analysing the representative sample. The sample itself will be completed through a nonrepeatable selection of units.

The object of this experimental study is the analysis of the degree of awareness and implementation of benchmarking in the Bulgarian manufacturing practice in medium and large companies (according to the number of staff) and situated geographically in North Central region, so this is the general aggregate to be studied.

\section{Identifying the general aggregate}

To identify the units in the general aggregate, which is the object of this statistical study, the authors will use the report of the National Statistical Institute (NSI) of the Republic of Bulgaria, published on 29.11.2019 (NSI, 2018). According to this report, in 2018, in North Central region the total number of companies was 36415 active companies, of which 3717 operate in the field of manufacturing. Besides the criterion for ongoing manufacturing activity, the companies to get in the general aggregate should meet one more requirement, namely, the size of the company according to the number of employees. According to NSI's report in 2018 there were 560 medium and large companies in North Central region. From the data mentioned so far, we can conclude that the general aggregate of this empirical study includes 560 units - large and medium companies in North Central region.

\section{Determining the size of the representative sample}

A sample is representative, when the units from it are studied exclusively while the results from their observation are extrapolated on the whole aggregate of units, i.e. they are summarised for the whole aggregate. The sample should meet two conditions simultaneously, so it can be considered representative in terms of mathematical statistics, and these are:

- the selection of those units that are included in the sample should be made randomly, and

- the units in the sample should be enough for the necessary size of the sample.

In order for samples to meet the requirements for representativeness in terms of applied statistics and, particularly, in terms of social and economic studies, the first condition should be changed. In this case, a sample should be considered representative if it meets simultaneoulsly the following two conditions:

- the characteristics and proportions of the sample should coincide with the main characteristics and proportions of the general aggregate;

- the sample units should form a large enough size of the sample.

This allows the principle of selecting the units in the sample to be intentional and not random, as long as the above-mentioned two conditionce are fulfilled.

The objective of each representative study is to obtain results with satisfactory accuracy. That is why as early as the stage of planning a given statistical survey, it is necessary for the organisers to know more or less the approximate accuracy of the results. At this stage it is necessary to determine thr size of the possibly maximum error at a certain level of warranty probability. When the organisers work with normal distribution and large enough samples, the so called warranty multiplier is used and its value is reported in a table for normal distribution at the accepted level of trusted probability. Practically, in specific studies, the most common warranty probability used is 0.95 where the warranty multiplier is 1.96 , or warranty probability 0.99 , where the warranty multiplier is 2.58. For example, using warranty probability 0.95 the risk of error is 0.05 , or of 100 samples the error will be in 5 , while for probability of 0.99 , only 1 of all 1000 will be wrong. It should be noted that the diminishing risk leads to expanding the trust interval (Kostov \& Tododrov, 1999).

In this study, to achieve the goals set, the authors will conduct partial statistical study through studying the representative sample, created by nonrepeatable selection of units.

The size of the sample is formed after the following formula for calculating the size of te representative smaple (Kostov, \& Todorov, 1999): 


$$
n=\left\{\frac{Z^{2} \cdot \sigma^{2} N}{\Delta^{2} N+Z^{2} \cdot \sigma^{2}}\right\},
$$

where: $n$ - sample size, $N$ - aggregate size, $Z-$ warranty multiplier, $\Delta-$ maximum permissible error size, $\sigma$-standard deviation.

In the specific case we can substitute the values as follows:

- $N$ - the size of the aggregate is already known from NSI'd report (NSI, 2018), namely 560 companies.

- $Z$ - the authors have previously chosen to work with warranty probability 0.95 , therefore $Z=1.96$ (the value has been determined by a table for normal distribution).

- $\Delta-$ the size of the maximum permissible error, determined by the authors at the beginning of the research is $15 \%$ or 0.15 .

- $\sigma-$ at the start of the study the standard deviation is unknown. That is why the authors accept 0.5 as the value of the standard deviation, or $50 \%$, which is the maximum value of this deviationis the maximum deviation value at a relative share.

The data already determined can be substituted in the formula (1) for calculating the size of the representative sample. It looks like this:

$$
n=\left\{\frac{1.96^{2} \times 0.5^{2} \times 560}{0.15^{2} \times 560+1.96^{2} \times 0.5^{2}}\right\}=39.66 .
$$

Therefore, in order for the sample to be fairly accurate and sufficiently reliable, as well as meeting the requirements for size, it should consist of 40 units, in this case, 40 manufacturing medium and large companies from North Central region.

Keeping the two conditions of authenticity of the representative sample and using the intentional selection, the authors identify as object of the experimental research medium and large companies (according to the number of staff), which according to the National classificator of economic activities (NCEA, 2019) are registered in sector C Manufacturing Industry and operate in the following sections:

- "Manufacturing of food product";

- "Manufacturing of clothes";

- "Manufacturing of chemicals";

- „Manufacturing of rubber and plastics“;

- "Manufacturing articles of other nonmetallic mineral raw materials";

- "Manufacturing of basic metals";
- "Manufacturing of metallic articles without machinery and equipment";

- "Manufacturing of computer and communication equipment, electronic and optical products";

- "Manufacturing of general and special purpose machines and equipment";

- "Manufacturing of automobiles and their engines";

- "Nonclassified manufacturing".

When selecting the objects the requirement for size of the company has been followed (according to number of employees), geographic principle (North Central region) and the presence of manufacturingactivity. The wide range of companies operating in the 11 sections of the sector Manufacturing Industry has been imposed, so that the conclusions from the analysis of the data collected could be valid for the whole aggregate.

\section{Survey - stage two}

Our own quantitative study has been conducted through a survey, used for collecting data. The survey has four sections. The questions are aimed at the company in general, and not at one of its separate business units. The total number of subquestions, which are part of the survey is 47 . The questionnaire contains both open answer (24), and closed answer (23) questions, some of which are presented as tables.

The survey envisaged analyses the awareness and implementation of benchmarking, as well as the factors and their values, critical for the organisation and determining the degree of necessity for changes, i.e. innovations.

The target group (survey respondents) are owners, directors and managers of medium and large companies (according to the nmber of employees).

The survey, conducted by the authors, consists of four sections with 47 questions to fill in of the type: one possible choice from many, several possible choices from a set of possible answers, filling in an open answer in a text field. For the answer to the so called "closed" questions, the metod for selecting the closest/correct answer has been used.

\section{The structure of the survey is as follows:}

Section I: its aim is to provide information about the specific respondent, namely, hiyerarchy rank, experience, education, etc. Also in this section we seek information about the company itself, presented by the respondent such as size 
(according to the number of employees), sphere of operation, competitors, market, etc. Some of the questions from Section I "Information part" are:

$$
\begin{aligned}
& \text { 1. Personal data } \\
& \text { 1.1. Occupation } \\
& \text { - senior manager; } \\
& \text { - medium level manager; } \\
& \text { - core personnel; } \\
& \text { - administrative personnel; } \\
& \text { - support personnel. } \\
& \text { 1.2. Department: } \\
& \text { - managing; } \\
& \text { - administrative; } \\
& \text { - trade; } \\
& \text { - manufacturing; } \\
& \text { - support; } \\
& \text { - other. }
\end{aligned}
$$

Section II is dedicated to benchmarking, knowing and implementing it, types of benchmarking used and the results from its use:

1. Has your company conducted a benchmarking survey:

Yes, once;

Yes, more than 3 times;

Yes, quite often;

Yes, this is part of the company's programme;

No, never.

2. What kind of benchmarking has been conducted:

Internal;

Competitive;

Strategic;

I can't answer.

Section III is called "Innovation strategy" and aims to find out how the innovation strategy is developed in the company and whether the benchmarking surveys (if there are any) are used in developing innovation strategies:

Section III Innovation strategy: please, mark your answer with a tick

1. Is there an Innovations Department in your company?

- No;

- Yes, but its functions and responsibilities are not clear;

- Yes, with clearly expressed functions and responsibilities.
5. Are the conducting of benchmarking surveys and analysing the results related to the innovation strategy:

- Yes;

- No;

- Not always;

- I am not sure.

\section{Semi-structured interview (method of expert evaluation)}

For the needs of this study, a semi-structured interview has been conducted with owners, and managers of medium and large size companies (according to the number of emplyees they have). The semi-structured or so called in-depth interview aims at in-depth collection of different types of information in personal contact with the respondents. This personal contact makes it possible to expand the field of information awareness of the interviewer to unsuspecting directions, which can point to a new problem. At the same time, it is valid only for the individual interviewee, and can be viewed only in the context of what is said by him/her. A serious drawback of this kind of survey is the time it takes, both in terms of the interview itseld and the processing and summarising of data (Lapinskiene, Peleckis, \& Slavinskaite, 2017). Despite these flaws, for the purpose of this study, such an interview was conducted with employees in key management positions, managers and owners of target group companies on key issues related to benchmarking research, the results from them, innovation strategies development and critical values of key performance indexes. This survey method has been chosen due to its flexibility, adaptability and sensitiveness to the topics (data related to the company performance, whose complexity is not available to all levels of management).

The main questions included in the interview are related mostly to the understanding of the benefits from benchmarking analysis, its implementation in developing innovation strategies and the critical values of performance indexes, which lead to the need for urgent innovations in the performance (Lindberg et al., 2015). The companies are diverse, but they all meet the limitations, namely, geographic - North Central region, medium and large companies, according to the number of employees and manufacturing activity.

The main questions in the structured interview are:

- How useful are the results from the benchmarking research? (Daunfeldt, Elert, \& Johansson, 2014). 
- Could the data obtained from the benchmarking analysis be implemented in developing innovations and consequently, serve as the foundation of developing an innovation strategy of the company? (Effendi et al., 2008).

- What is the process of developing an innovation strategy? (Mihailova, 2008).

- Based on the developed plans/budgets, please, indicate which activity indexes and deviations in their budget values are critical and show the need for immediate changes? (Antosova et al., 2013).

The duration of each interview is between thirty minutes and one hour and thirty minutes, depending on the answers and the interest demonstrated on the part of the interviewed.

\section{Conclusions}

As a resalts of literature review authors found out that there is not enough or missing an information at all about empirical study regarding the benchmarking and a methodology for its implementation. This necessitates the development of a methodology for conducting empirical research aimed at demonstrating the extent to which benchmarking is known and used as an approach for innovative management in Bulgarian practice. The result from the methods for conducting an empirical study applied and the compliance with all principles and conditions led to the collection of the primary data needed. The data collected from the sample defined above are satisfactorily accurate and present the characteristics, summarised for the whole general aggregate. The processing and analysis of the data collected will follow and the conclusions made will be the basis for developing a concept for implementing benchmarking as an approach to innovation management.

\section{References}

Antosova, M., Csikosova, A., Culkova, K., \& Senova, A. (2013). Benchmarking research of steel companies in Europe. Metalurgija - Sisak Then Zagreb, 52(3), 410-412.

Boneva, M. (2018). Challenges related to the digital transformation of business companies. In Proceedings of the 6th international conference innovation management, entrepreneurship and sustainability (pp. 101-114).

Chiprianov, M. (2008). Product-oriented benchmarking research, Dialogue Magazine, 3, 1-26.

Chengelova, E. (2013). A holistic approach to the study of shadow economy. National Archives Magazine,
CA "D. Tsenov ", Svishtov, book. 1/2013 (subprinting) (pp. 159-180).

Conev, V. (1968). Basics of representative research. Sofian.

Daunfeldt, S. O., Elert, N., \& Johansson, D. (2014). The economic contribution of high-growth firms: do policy implications depend on the choice of growth indicator? Journal of Industry, Competition and Trade 14(3), 337-365.

https://doi.org/10.1007/s10842-013-0168-7

Effendi, M., Razali, M. M., Rohana, A., \& Adi, S. (2008). A Study on the development of Key Performance Indicators (KPI) at an aerospace manufacturing company. Journal of Advanced Manufacturing Technology, 2(2), 1-17.

Gatev, K. (1995). Introduction to statistics. Lia.

Ghinea, V., Mihailova, L., \& Papazov, E. (2015). Organizational cullture dynamics complex systems dynamics. Quality-access To Success, 16(147), 99-105.

Human Resources Development Operational Program. (2014-2020).

Kirova, M. (2009). Criteria for assessing the potential of innovation. Primex.

Kirova, M. (2011). Innovation management. Primex.

Kostadinova, I., \& Antonova, D. (2018). Key competencies in sustainability: assessment of innovative factors influencing the development of human resources in health care system proceedings. In the 6th International Conference Innovation Management, Entrepreneurship and Sustainability (pp. 577-590). Prague.

Kostov, S., \& Todorov, T. (1999). Statistics: general theory. AI Svishov.

Lapinskiene, P., \& Slavinskaite, N. (2017). Energy consumption, economic growth and greenhouse gas emissions in the European Union countries. Journal of Business Economics and Management, 18 (6),1082-1097. https://doi.org/10.3846/16111699.2017.1393457

Lindberg, C., Tan, S., Yan, J., \& Starfelt, F. (2015). Key performance indicators improve industrial performance. Energy Procedia, 75, 1785-1790. https://doi.org/10.1016/j.egypro.2015.07.474

Marinov, M., Vitliemov, P., \& Popova, E. (2017). Towards Big Data and internet of things as key aspects of energy efficiency. TEM Journal Technology Education Management Informatics, 6(3), 427-435.

Mihailova, L. (2008). Highlights of the study and analysis of the companies' activities and their business consulting. In Satistical Reports of the International Scientific Conference on Companies and Markets in Bulgaria in the Context of European Integration - Continuing Adaptation (pp. 431-435). YI - Varna.

Nedyalkov, A. (2011). Methodology for measuring the quality of services. In IX International Scientific 
Conference "Management and Engineering" (pp. 228-237). Sofia.

NSI. (2018). https://www.nsi.bg/bg

Pavlov, V. (2013). Applied statistics. Prepress.

Pavlov, D., Sheresheva, M., \& Perello, M. (2017). The intergenerational small family enterprises as strategic entities for the future of the European civilization - a point of view. Journal of Entrepreneurship \& Innovation, 3, 26-38.
Saikova, I., \& Todorova, S. (1994). The statistical survey. The New Bulgrian University.

Simeonova, A., \& Nedyalkov, A. (2019). A Priori Research on Lean Tools in Business). In Proceedings of University of Ruse - 2019, Vol. 58, book 5.1. Ruse, University of Ruse. ISBN 1311-3321.

The Global Benchmaking Network. (2010). Global survey on business improvement and benchmarking. 